\title{
Diagnostic odyssey of patients with mitochondrial disease
}

\section{Results of a survey}

Johnston Grier, MPH, Michio Hirano, MD, Amel Karaa, MD, Emma Shepard, BS, and John L.P. Thompson, PhD

Neurol Genet 2018;4:e230. doi:10.1212/NXG.0000000000000230

\section{Abstract}

\section{Objective}

To document the complex "diagnostic odyssey" of patients with mitochondrial disease.

\section{Methods}

We analyzed data from 210 Rare Diseases Clinical Research Network Contact Registry participants who were patients with a biochemical deficiency or self-reported diagnosis of mitochondrial disease, or their caregivers.

\section{Results}

Participants saw an average of 8.19 clinicians (SD 8.0, median 5). The first clinician consulted about symptoms was typically a primary care physician (56.7\%), although $35.2 \%$ of participants initially sought a specialist. Of note, $55.2 \%$ of participants received their diagnosis from a neurologist, $18.2 \%$ from a clinical geneticist, and $11.8 \%$ from a metabolic disease specialist. A majority of the participants $(54.6 \%)$ received 1 or more nonmitochondrial diagnoses before their final mitochondrial diagnosis. In their pursuit of a diagnosis, $84.8 \%$ of participants received blood tests, $71 \%$ a muscle biopsy, $60.5 \% \mathrm{MRI}$, and $38.6 \%$ urine organic acids. In addition, $39.5 \%$ of the participants underwent mitochondrial DNA sequencing, 19\% sequencing of nuclear gene(s), and $11.4 \%$ whole-exome sequencing.

\section{Conclusions}

The diagnostic odyssey of patients with mitochondrial disease is complex and burdensome. It features multiple consultations and tests, and, often, conflicting diagnoses. These reflect disease variety, diagnostic uncertainty, and clinician unfamiliarity. The current survey provides an important benchmark. Its replication at appropriate intervals will assist in tracking changes that may accompany increased popularity of exome testing, more rigorous diagnostic criteria, increased patient reported outcome activity, and trials for promising therapies.

\author{
Correspondence \\ Dr. Thompson \\ jlt12@cumc.columbia.edu
}




\section{Glossary}

IRB = institutional review board; NAMDC $=$ North American Mitochondrial Disease Consortium; PCP $=$ primary care physician; RDCRN = Rare Diseases Clinical Research Network; WES = whole-exome sequencing.

Mitochondrial diseases comprise a group of rare, about 1 in 4,300, debilitating genetic disorders. ${ }^{1}$ They are due to defects affecting the mitochondrial oxidative phosphorylation pathway and are caused by genetic mutations in the mitochondrial genome, nuclear DNA, or both. ${ }^{2}$ The disorders feature a wide array of multisystemic manifestations that frequently include developmental abnormalities or regression, myopathy, seizures, dementia, hearing loss, blindness, strokes, diabetes mellitus, and premature death affecting both children and adults. Most mitochondrial diseases are progressive and have poor prognoses. There are few available treatments. ${ }^{2}$

Mitochondrial diseases are difficult to diagnose reliably, because of their wide clinical and genetic heterogeneity, and require a detailed medical history and extensive knowledge and expertise on behalf of the diagnosing physician. Anecdotal evidence from clinicians, researchers, and patients suggests that before finally receiving a diagnosis of mitochondrial disease, many patients go through a long ordeal, visiting numerous clinical specialists, receiving other conflicting diagnoses, and enduring repeated and sometimes invasive testing. In addition, misdiagnoses are not uncommon. The increasing ubiquity and rapidly falling cost of genetic testing such as whole-exome sequencing (WES), coupled with a growing understanding of the genetic origins of mitochondrial diseases, are changing how patients receive diagnoses of mitochondrial diseases. To date, however, research on mitochondrial diseases, including recruitment of patients for research studies and clinical trials, has been hampered by their low incidence, heterogeneity, and underdiagnosis by clinicians.

The goal of this study was to increase the understanding of this "diagnostic odyssey" by a survey of patients with selfreported mitochondrial disease.

\section{Methods}

\section{Survey population}

Respondents were enrollees in the established Patient Contact Registry of the Rare Diseases Clinical Research Network (RDCRN), an initiative of the NIH Office of Rare Disease Research. The survey was designed and analyzed by investigators in the North American Mitochondrial Disease Consortium (NAMDC), an RDCRN member. It was administered over 2.5 months between mid-October 2015 and early January 2016. Institutional review board (IRB) approval was obtained by the RDCRN Data Management and Coordinating Center at the University of Southern Florida in Tampa, FL. Eligible individuals were RDCRN Contact
Registry participants with a self-reported diagnosis of mitochondrial disease, or their caregivers, who had an email address, were able to provide informed consent, and were capable of completing the survey. Respondents enrolled online through a link on the NAMDC public website (rarediseasesnetwork.org/namdc).

\section{Data collection}

Participation was solicited through an email invitation describing the purpose and length of the survey, which was sent to all RDCRN-NAMDC Contact Registry participants with email addresses. Each email included a unique direct link, which ensured that the participant's survey responses could be linked to basic demographic data they had previously provided to the RDCRN Registry. Participants entered data directly online using encrypted communication links. Participants were asked to contact the principal investigator (J.G.), whose contact information was provided in the email, with questions. A follow-up email with the same content was sent to eligible participants who had not completed the survey 1 month after data collection began.

The survey (appendix e-1, lww.com/NXG/A41) consisted of 16-25 questions, depending on skip patterns. The estimated average completion time was 15 minutes. Wherever possible, the survey used questions that required yes or no answers or dropdown boxes and radio buttons and included an "other" option permitting a free text answer. Data quality and consistency were maintained by strict monitoring and constraints at data entry.

\section{Standard protocol approvals, registrations, and patient consents}

Informed consent was obtained and documented online using an IRB-approved electronic consent form, which confirmed that participants were free to decline entry into the survey or to withdraw at any time without prejudice to future treatment. To avoid linking protected health information to the study data, participants' names were not collected; only individuals' agreements to participate were recorded. IRB approval for the research was provided by the University of Southern Florida in Tampa, FL. No personal communications are cited in the article. The RDCRN study number for the survey is 7,352 .

\section{Specific aims}

The survey was designed to address 6 prespecified questions:

1. How much time typically elapses between when patients initially notice symptoms of a mitochondrial disorder and when they receive the diagnosis of mitochondrial disease?

2. How many physicians do patients typically see before they receive the diagnosis of mitochondrial disease? 
3. What testing do patients typically undergo?

4. What other diagnoses, if any, do patients typically receive?

5. How did receiving a mitochondrial disease diagnosis alter the patient's life?

6. If the patient were to learn that their mitochondrial disease diagnosis was incorrect, what effect would that have?

We did not collect results of molecular genetic testing because of inaccuracies in the interpretation of mutations (e.g., single heterozygous autosomal recessive mutations were sometimes considered to be the cause of patient's disease).

\section{Analysis}

Results are reported as frequency counts with percentages. The data were analyzed using IBM SPSS Statistics version 22 (Armonk, NY), and all results were validated independently using SAS version 9.4.

\section{Results}

Two hundred fifteen participants completed the survey. Five failed to indicate either a clinical diagnosis or a biochemical deficiency and were excluded, leaving 210 for analysis. Of these, $73(34.8 \%)$ reported both a biochemical deficiency and a clinical diagnosis; 134 (63.8\%) only a clinical diagnosis; and 3 (1.4\%) only a biochemical deficiency. Molecular genetic data were not specifically collected and were not provided by participants. Seventy-eight respondents (37.1\%) were caretakers, and 132 $(62.9 \%)$ were patients who took the survey for themselves.

Table 1 presents the breakdown of self-reported mitochondrial disease diagnoses. The most common was myopathy (21.8\%), followed by chronic progressive external ophthalmoplegia (9.6\%), and mitochondrial encephalomyopathy, lactic acidosis, and stroke-like episodes (9.1\%). Fifty-six participants (28.4\%) reported "Other" mitochondrial diseases. The diseases entered by these participants were various, and while some were known mitochondrial diseases not available in the drop-down list, the majority could be categorized as biochemical deficiencies, other mitochondrial nuclear gene defects, or other metabolic diseases with secondary mitochondrial dysfunction.

The most commonly reported symptom that motivated patients to see a doctor was weakness (62.4\% of participants), followed by fatigue (56.2\%), difficulty walking (38.6\%), droopy eyelids (32.9\%), and impaired coordination (32.4\%). Table 2 provides a complete list. One hundred nineteen participants $(56.7 \%)$ reported first discussing their mitochondrial disease symptoms with their primary care physician (PCP), and 74 (35.2\%) with a specialist (17 [8.1\%] either did not answer the question or reported first discussing their mitochondrial disease symptoms with neither a PCP nor a specialist).
Table 1 Self-reported mitochondrial disease diagnosis

\begin{tabular}{ll}
\hline Self-reported disease & Frequency (\%) \\
\hline Myopathy & $43(21.8)$ \\
\hline CPEO & $19(9.6)$ \\
\hline MELAS & $18(9.1)$ \\
\hline Leigh syndrome & $16(8.1)$ \\
\hline Kearns-Sayre syndrome & $9(4.6)$ \\
\hline Encephalomyopathy & $8(4.1)$ \\
\hline LHON & $6(3.0)$ \\
\hline CPEO-plus & $5(2.5)$ \\
\hline Multiple systemic syndrome & $5(2.5)$ \\
\hline NARP & $4(2.0)$ \\
\hline MERRF & $3(1.5)$ \\
\hline Alpers syndrome & $2(1.0)$ \\
\hline SANDO & $2(1.0)$ \\
\hline Encephalopathy & $1(0.5)$ \\
\hline Other & $56(28.4)$ \\
\hline Total & $197(100)$ \\
\hline
\end{tabular}

Abbreviations: $\mathrm{CPEO}=$ chronic progressive external ophthalmoplegia; $\mathrm{LHON}=$ Leber hereditary optic neuropathy; MELAS = mitochondrial encephalomyopathy, lactic acidosis, and stroke-like episodes; MERRF = myoclonic epilepsy with ragged-red fibers; NARP = neuropathy ataxia retinitis pigmentosa; SANDO = sensory ataxic neuropathy, dysarthria, and ophthalmoplegia.

${ }^{a}$ Thirteen participants with missing or unsatisfactory answers are excluded.

Only 27 respondents (12.9\%) reported that the doctor with whom they first discussed their symptoms was the physician who provided them with a mitochondrial disease diagnosis. Among the 199 participants who answered the question "Including the doctor with whom you first discussed your symptoms and the doctor who diagnosed your mitochondrial disease, how many doctors did you consult?", the average number of doctors consulted was $8.19(\mathrm{SD}=8.0$, median $=5)($ table 3$)$.

Among patients who reported receiving their mitochondrial disease diagnosis from a specialist, $112(55.2 \%)$ received their diagnosis from a neurologist (table 4). The next most common diagnosing specialists were clinical geneticists $(18.2 \%)$ and metabolic disease specialists (11.8\%) (table 4$)$.

One hundred seventy-eight participants $(84.8 \%)$ reported having blood testing as part of the process of receiving their mitochondrial disease diagnosis. Among these, 116 reported tests for blood lactate, 97 for pyruvate, 103 for amino acids, and 84 reported that their blood was used for genetic testing. Other common tests were muscle biopsies (71\%), MRI $(60.5 \%)$, and urine organic acids $(38.6 \%)$.

Eighty-three participants (39.5\%) reported receiving mitochondrial DNA sequencing as part of the process of receiving 
Table 2 Self-reported motivation for initial consultation with a doctor

\begin{tabular}{ll}
\hline Symptom & Frequency (\%) \\
\hline Weakness & $131(62.4)$ \\
\hline Fatigue & $118(56.2)$ \\
\hline Difficulty walking & $81(38.6)$ \\
\hline Other & $71(33.8)$ \\
\hline Droopy eyelids & $69(32.9)$ \\
\hline Impaired coordination & $68(32.4)$ \\
\hline Numbness/weakness in the hands/feet & $67(31.9)$ \\
\hline Gastrointestinal discomfort & $62(29.5)$ \\
\hline Developmental delay & $49(23.3)$ \\
\hline Seizures & $39(18.6)$ \\
\hline Loss of vision & $31(14.8)$ \\
\hline Hearing loss & $27(12.9)$ \\
\hline Diabetes & $10(4.8)$ \\
\hline Heart disease & $6(2.9)$ \\
\hline Kidney disease & $5(2.4)$ \\
\hline Liver disease & $3(1.4)$ \\
\hline
\end{tabular}

a Percentages calculated of 210 respondents. Participants could indicate more than 1 symptom. All indicated at least 1.

their mitochondrial disease diagnosis. Forty (19\%) reported undergoing nuclear DNA gene sequencing, and only 24 (11.4\%) reported having WES.

One hundred seventy participants $(81.7 \%)$ reported beginning a treatment management plan after receiving a mitochondrial disease diagnosis. One hundred fifty (71.8\%) reported joining a patient support group, patient advocacy group, or other sort of community for individuals with mitochondrial disease as a result of their diagnosis. Among those

Table 3 Number of doctors consulted before diagnosis

\begin{tabular}{ll}
\hline No. of doctors & Frequency (\%) $^{\mathbf{a}}$ \\
\hline $\mathbf{1 - 5}$ & $102(52.0)$ \\
\hline $\mathbf{6 - 1 0}$ & $46(23.5)$ \\
\hline $\mathbf{1 1 - 1 5}$ & $21(10.7)$ \\
\hline $\mathbf{1 6 - 2 0}$ & $20(10.2)$ \\
\hline $\mathbf{2 0}$ & $7(3.6)$ \\
\hline Total & $196(100)$
\end{tabular}

${ }^{\text {a }}$ Fourteen participants with missing or unsatisfactory answers are excluded.
Table 4 Specialty of the diagnosing physician

\begin{tabular}{ll}
\hline Specialty & Frequency (\%) \\
\hline Neurology & $112(55.2)$ \\
\hline Clinical genetics & $37(18.2)$ \\
\hline Metabolic disease & $24(11.8)$ \\
\hline Ophthalmology & $14(6.9)$ \\
\hline Other & $8(3.9)$ \\
\hline Rheumatology & $5(2.5)$ \\
\hline Immunology & $2(1.0)$ \\
\hline Endocrinology & $1(0.5)$ \\
\hline Total & $203(100)$ \\
\hline
\end{tabular}

${ }^{a}$ Seven participants with missing or unsatisfactory answers are excluded.

who reported joining a patient community, $84.7 \%$ reported that they felt participation or membership in such a community was beneficial for them.

One hundred thirteen participants $(54.6 \%)$ reported receiving an alternative nonmitochondrial disease diagnosis before ultimately receiving their mitochondrial disease diagnosis; and of these, $31.9 \%$ received more than 1 . The most common nonmitochondrial diagnoses are summarized in table 5 . The most frequently reported motivating factor for seeking a different diagnosis after receiving a nonmitochondrial disease diagnosis was that the treatment the participant was receiving for their disease symptoms did not help them (reported by 59 participants).

Table 5 Common nonmitochondrial diagnoses

\begin{tabular}{ll}
\hline Diagnosis & $\begin{array}{l}\text { Frequency } \\
(\%)^{\mathrm{a}}\end{array}$ \\
\hline $\begin{array}{l}\text { Psychiatric disorder (depression, conversion } \\
\text { syndrome, etc.) }\end{array}$ & $15(13.3)$ \\
\hline Fibromyalgia & $13(11.5)$ \\
\hline Chronic fatigue syndrome & $10(8.8)$ \\
\hline Multiple sclerosis & $9(8.0)$ \\
\hline $\begin{array}{l}\text { Gastrointestinal disease (irritable bowel } \\
\text { syndrome, gastroparesis, etc.) }\end{array}$ & $8(7.1)$ \\
\hline Seizure & $8(7.1)$ \\
\hline Myasthenia gravis & $7(6.2)$ \\
\hline Rheumatological & $7(6.2)$ \\
\hline Chronic pain & $6(5.3)$ \\
\hline
\end{tabular}

a Percentages calculated of 113 participants who received a nonmitochondrial disease diagnosis. 
Finally, participants were asked: "If you were to learn today that the mitochondrial disease diagnosis you received is incorrect, and that you do not in fact have a mitochondrial disease, how would this affect you?" Among the 201 who responded to this question, $23(11.4 \%)$ answered that it would affect them very negatively, 42 (20.9\%) negatively, 60 (29.9\%) not at all, 45 (22.4\%) positively, and 31 (15.4\%) very positively. The number of physicians consulted among the 65 participants who expected negative or very negative feelings was lower than it was among the 136 participants who did not expect negative feelings after a mistaken diagnosis (median 6.5 vs 5.0 physicians consulted, $p=0.086$ by 2 -sided Wilcoxon rank-sum test, since the number of physicians is not normally distributed). This 1.5 physician difference is not significant at the conventional 0.05 level, but is at the exploratory 0.10 level. We consider this result suggestive, given the relatively small Ns, and worth further investigation.

\section{Discussion}

The results highlight the difficulty, confusion, and burden that individuals with suspected mitochondrial disease typically undergo in obtaining a diagnosis. While the survey failed to capture the amount of time that typically elapses between the symptom onset and diagnosis, it is clear from several answers that the process of obtaining a mitochondrial disease diagnosis can frequently take many years and multiple doctors (mean 8.19, median 5). There are multiple reasons for this diagnostic delay, such as the physician's unfamiliarity with the diseases due to their rarity, the heterogeneity of the symptoms that are difficult to merge into a unifying diagnosis, and the wide range of initial symptoms that tend to be nonspecific. All these factors contribute to the high proportion of participants who received other nonmitochondrial diagnoses (54.3\%) before their final diagnoses.

The variety and sheer amount of testing reported by participants is a further reflection of the difficulty of mitochondrial disease diagnosis. Of particular note, $71 \%$ of patients reported receiving a muscle biopsy. Until a few years ago, this painful and invasive procedure was the most reliable way to obtain a definitive diagnosis through biochemical and histologic testing. Evidence indicates that clinicians and researchers today are moving away from biochemical testing and relying more on molecular genetic testing. ${ }^{3}$ This is especially true as a rapidly expanding number of genetic determinants of mitochondrial diseases, both in mitochondrial and nuclear DNA, are being identified. ${ }^{2}$ Planned future surveys will help track changes in testing practices and will hopefully identify reductions in invasive testing, such as muscle biopsy, and increased genetic testing, especially whole-exome and whole mitochondrial DNA sequencing. ${ }^{4}$

The survey confirmed considerable anecdotal evidence from patients and clinicians that individuals with mitochondrial disease have often received incorrect diagnoses.
It is perhaps not surprising, given how poorly understood mitochondrial diseases are, that the most common nonmitochondrial diagnoses were psychiatric disorders such as depression and conversion. This further highlights the unfamiliarity with the diagnosis by health care providers and the skepticism in recognizing several nonspecific, seemingly unrelated symptoms as worth investigating. Manifestations of other reported nonmitochondrial diagnoses fit perfectly with common symptoms of mitochondrial diseases and were also not surprising. These include fibromyalgia; Lyme disease; and chronic fatigue syndrome, which produce manifestations that resemble the reduced energy state of patients with mitochondrial disease; MS, which produces neurologic manifestations and fatigue; and gastrointestinal diseases, which cause cachexia and reduced gastrointestinal motility that are often present in patients with mitochondrial disease. ${ }^{5}$

The effect of a mitochondrial disease diagnosis on participants proved hard to quantify. Most patients began a treatment management plan as a result of their diagnosis, ${ }^{6}$ although about one-fifth did not. This lack of therapeutic intervention is largely due to the fact that few disease-modifying treatments are available. ${ }^{7}$ In addition, $28.6 \%$ of participants did not report joining a patient support group or community group of any kind. This is in line with a previous survey showing that the major support systems for patients include their families and health care providers. ${ }^{8}$

Participants were almost evenly divided in their responses to the question, "If you were to learn today that the mitochondrial disease diagnosis you received is incorrect, and that you do not in fact have a mitochondrial disease, how would this affect you?" As with the answers regarding a treatment management plan, this could reflect the lack of available treatments for mitochondrial diseases. Since a diagnosis of mitochondrial disease often does not lead to a specific treatment, especially one that is likely to control symptoms and improve quality of life, perhaps participants ultimately found their diagnosis irrelevant. In a prior mitochondrial disease survey, ${ }^{8}$ patients and families with genetically confirmed and nonconfirmed mitochondrial disease perceived problems with their medical and social support. Those with a confirmed genetic diagnosis consistently perceived themselves to be better off than those patients without a confirmed diagnosis, highlighting the emotional stress and anxiety of having a serious undiagnosed problem and feeling disadvantaged within the medical community by not receiving the care they require.

A potential explanation of our provisional finding that participants who have seen more physicians may be more likely to be negatively affected by learning that their diagnosis is incorrect is that participants who have visited more doctors to reach their mitochondrial disease diagnosis have spent more effort searching for answers and are more invested in their current diagnosis. Learning that this is incorrect and that they 
will have to visit yet another doctor could certainly be very disheartening.

This survey provided quantitative data confirming the longestablished view of many patients with mitochondrial disease, researchers, and clinicians that mitochondrial disease diagnosis is difficult, confusing, and burdensome. Clinicians and researchers are making strides to combat this problem, such as developing clear and widely used diagnostic criteria, ${ }^{2,3,9}$ and perfecting genetic testing. ${ }^{4,10}$ Patients with mitochondrial disease, through Patient Advocacy Groups or as individuals, are raising awareness of these diseases. Yet, because of the rarity and diffuse nature of mitochondrial diseases, the difficulty of diagnosis is not likely to go away any time soon. The situation may change when genetic testing is more widely used by health care systems in place now or in the future. Given these developments, it is important to continue to track changes in the odyssey of patients with mitochondrial disease; to advance efforts to reduce the burden on patients; and to include the links between genetic tests and congruent phenotypes in future work.

\section{Strengths and limitations}

Strengths of the survey include the attempts to keep the number of questions to the minimum necessary to achieve the survey's aims and to craft clear, concise, closed-ended questions, all of which to improve data quality. ${ }^{11,12}$

The primary weakness is the unreliability of self-reported data. Attempts to minimize this included using closed-ended yes or no questions; restricting other answers by the use of dropdown lists; and careful wording to make questions as clear and unambiguous as possible.

Another issue is that we cannot be certain that participants actually have the mitochondrial diseases they believe they have. One strategy to address this was to exclude patients who did not specifically affirm that they had been informed by a health professional that they had a mitochondrial disease or that they had a biochemical deficiency. Furthermore, this problem is somewhat limited. Whether an individual respondent actually has a mitochondrial disease is irrelevant with respect to the initial stages of the patient odyssey. That the respondent initially thinks that they may have a mitochondrial disease is critical. At the end, we cannot be sure that the diagnosis is correct. But this is a problem in the field in general, which we hope the development and application of rigorous diagnostic criteria by NAMDC will alleviate. ${ }^{13}$ By applying these criteria, which include clinical, histologic, biochemical, and molecular genetic data, we will obtain more accurate information about the diagnostic odyssey of patients with definite mitochondrial diseases.

Further detailed analysis of the data was difficult because of the many combinations of answers provided by the respondents For example, 210 participants reported more than 800 symptoms. Given this, the impact of any individual symptom on an outcome such as number of physicians seen cannot be estimated statistically: it is confounded by the many combinations of other symptoms also displayed by the patients who present with it.

The survey failed to achieve its aim of determining the typical amount of time between the symptom onset and diagnosis of mitochondrial disease. This was an oversight. In retrospect, it would have been best to simply ask this question of the participants outright.

\section{Author contributions}

Johnston Grier: study concept and design, acquisition of data, interpretation of data, and drafting of the manuscript and tables. Michio Hirano and Amel Karaa: study concept and design, interpretation of data, and editing of the manuscript and tables. Emma Shepard: interpretation of data, statistical analysis, and editing of the manuscript. John L.P. Thompson: study concept and design, data interpretation, and editing and revision of the manuscript and tables.

\section{Acknowledgment}

The North American Mitochondrial Disease Consortium (NAMDC) is part of Rare Diseases Clinical Research Network (RDCRN), an initiative of the Office of Rare Diseases Research (ORDR), NCATS. This consortium is funded through collaboration between the NCATS and NIH. The NAMDC is jointly funded through an NIH U54 grant mechanism by the National Center for Advanced Translational Sciences (NCATS), the NINDS, the Eunice Kennedy Shriver National Institute of Child Health and Development (NICHD), and the Office of Dietary Supplements (ODS). In addition, the NAMDC receives private funding from the United Mitochondrial Disease Foundation (UMDF), a Patient Advocacy Group (PAG) for individuals with mitochondrial disease and their family members, and other private foundations. The NAMDC is part of the Rare Diseases Clinical Research Network (RDCRN), an initiative of the Office of Rare Disease Research (ORDR). The RDCRN consists of 22 research consortia, of which the NAMDC is one. The authors acknowledge the contributions of the patients and families who participated in this survey and of Amy Holbert, Health Informatics Institute/RDCRN, University of South Florida, for administrative support.

\section{Study funding}

This study was sponsored by the NIH under grant 5U54NS078059-06.

\section{Disclosure}

J. Grier is an employee of Bristol-Myers Squibb. M. Hirano has received travel funds from the American College of Medical Genetics; is a consultant of Meves Pharmaceuticals; has received honoraria for participating in advisory boards of Stealth BioTherapeutics Inc., Sarepta Therapeutics Inc., and Biogen; has received research support from NIH grants 1 U54 
NS078059-06 and P01 HD080642, Santhera Pharmaceuticals, Edison Pharmaceuticals, the Arturo Estopinan TK2 Research Fund, the Mileti Fund, the Nicholas Nunno Foundation, the Muscular Dystrophy Association (MDA) United States, and the Marriott Mitochondrial Disease Clinic Research Fund (MMDCRF); is listed as an inventor on a Columbia University patent application; and holds a patent for Rare pediatric disease (RPD) and orphan drug designations (ODD) for deoxynucleoside therapy for mitochondrial DNA depletion syndrome including TK2 deficiency. A. Karaa has served on the scientific advisory boards of MitoAction (nonprofit), the United Mitochondrial Disease Foundation (nonprofit), the National Fabry Disease Foundation (nonprofit), MitoBridge (commercial), and Sanofi Genzyme; has served on the editorial board of NEJM Knowledge+; has received travel funding/speaker honoraria from the United Mitochondrial Disease Foundation (nonprofit), the Fabry Support and Information Group (nonprofit), and Sanofi Genzyme (commercial); is a board member of the Mitochondrial Medicine Society (nonprofit); has received research support from the NIH; and has received grant support, consulting fees, and honoraria from Sanofi Genzyme; grant support and consulting fees from Shire and Stealth BioTherapeutics; consulting fees from Akros Pharma, Concert, Alexion, and Lumleian; and grant support from Reata Pharmaceuticals, Protalix BioTherapeutics; and Mitobridge. E. Shepard reports no disclosures. J. Thompson has received research support from NIH grants 1U54 NS078059-06 and P30 ES009089-15, FDA grant UFDSP00011270, and
Novartis Pharmaceuticals. Full disclosure form information provided by the authors is available with the full text of this article at Neurology.org/NG.

Received September 5, 2017. Accepted in final form December 27, 2017.

\section{References}

1. Gorman G, Schaefer A, Ng Y, et al. Prevalence of nuclear and mitochondrial DNA mutations related to adult mitochondrial disease. Ann Neurol 2015;77:753-759.

2. Gorman G, Chinnery P, DiMauro S, et al. Mitochondrial diseases. Nat Rev Dis Primers 2016;2:16080.

3. Parikh S, Goldstein A, Koenig MK, et al. Diagnosis and management of mitochondrial disease: a consensus statement from the Mitochondrial Medicine Society. Genet Med 2015;17:689-701.

4. Dare JT, Vasta V, Penn J, Tran NT, Hahn SH. Targeted exome sequencing for mitochondrial disorders reveals high genetic heterogeneity. BMC Med Genet 2013; 14:118.

5. Martikainen MH, Chinnery PF. Mitochondrial disease: mimics and chameleons. Pract Neurol 2015; 15:424-435.

6. Parikh S, Goldstein A, Koenig MK, et al. Practice patterns of mitochondrial disease physicians in North America. Part 1: diagnostic and clinical challenges. Mitochondrion 2014;14:26-33.

7. Moraes CT, Anderson V, Mohan C. Translational research in primary mitochondrial diseases: challenges and opportunities. Mitochondrion 2013;13:945-952.

8. Krieg E, Calderwood L, Campion MA, et al. Confirmed versus suspected: the social significance of a genetic or non-genetic diagnosis of mitochondrial disease. Mitochondrion 2016;28:60-66.

9. Parikh S, Goldstein A, Karaa A, et al. Patient care standards for primary mitochondrial disease: a consensus statement from the Mitochondrial Medicine Society. Genet Med 2017; 19. doi: 10.1038/gim.2017.107.

10. Karaa A, Rahman S, Lombès A, et al. Common data elements for clinical research in mitochondrial disease: a National Institute for Neurological Disorders and Stroke project. J Inherit Metab Dis 2017;40:403-414.

11. Galesic M, Bosnjak M. Effects of questionnaire length on participation and indicators of response quality in a web survey. Public Opin Q 2009;73:349-360.

12. Fan W, Yan Z. Factors affecting response rates of the web survey: a systematic review. Comput Hum Behav 2010;26:132-139.

13. North American Mitochondrial Disease Consortium. Available at: rarediseasesnetwork.org/cms/NAMDC. Accessed June 19, 2017. 


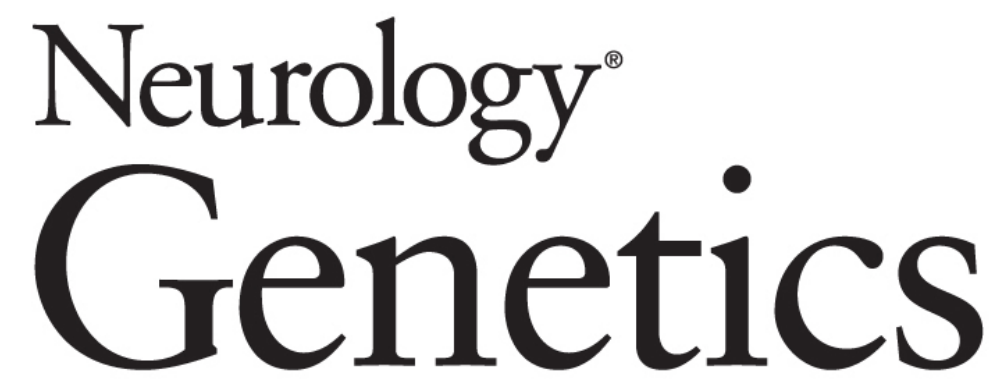

Diagnostic odyssey of patients with mitochondrial disease: Results of a survey Johnston Grier, Michio Hirano, Amel Karaa, et al. Neurol Genet 2018;4;

DOI 10.1212/NXG.0000000000000230

This information is current as of March 26, 2018

Neurol Genet is an official journal of the American Academy of Neurology. Published since April 2015, it is an open-access, online-only, continuous publication journal. Copyright Copyright $(2018$ The Author(s). Published by Wolters Kluwer Health, Inc. on behalf of the American Academy of Neurology.. All rights reserved. Online ISSN: 2376-7839.

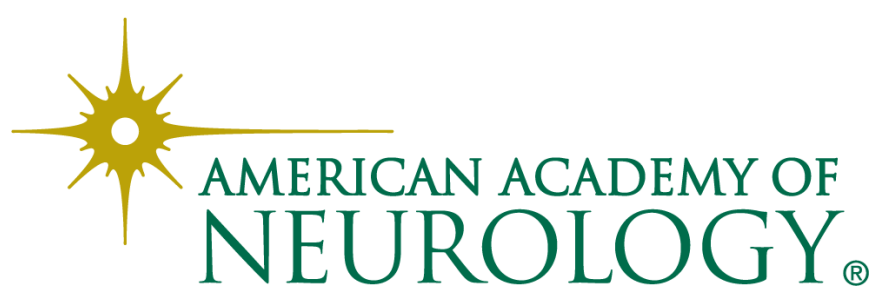




\section{Updated Information \& Services}

\section{Supplementary Material}

\section{References}

Citations

Subspecialty Collections

Permissions \& Licensing

\section{Reprints}

including high resolution figures, can be found at: http://ng.neurology.org/content/4/2/e230.full.html

Supplementary material can be found at: http://ng.neurology.org/content/suppl/2018/04/02/4.2.e230.DC1

This article cites 12 articles, 1 of which you can access for free at: http://ng.neurology.org/content/4/2/e230.full.html\#\#ref-list-1

This article has been cited by 7 HighWire-hosted articles: http://ng.neurology.org/content/4/2/e230.full.html\#\#otherarticles

This article, along with others on similar topics, appears in the following collection(s):

\section{Medical care}

http://ng.neurology.org//cgi/collection/medical_care

Metabolic disease (inherited)

http://ng.neurology.org//cgi/collection/metabolic_disease_inherited Mitochondrial disorders

http://ng.neurology.org//cgi/collection/mitochondrial_disorders Mitochondrial disorders; see Genetics/Mitochondrial disorders http://ng.neurology.org//cgi/collection/mitochondrial_disorders_see_ge netics-mitochondrial_disorders

Quality of life

http://ng.neurology.org//cgi/collection/quality_of_life

Information about reproducing this article in parts (figures,tables) or in its entirety can be found online at:

http://ng.neurology.org/misc/about.xhtml\#permissions

Information about ordering reprints can be found online: http://ng.neurology.org/misc/addir.xhtml\#reprintsus

Neurol Genet is an official journal of the American Academy of Neurology. Published since April 2015, it is an open-access, online-only, continuous publication journal. Copyright Copyright ( 2018 The Author(s). Published by Wolters Kluwer Health, Inc. on behalf of the American Academy of Neurology.. All rights reserved. Online ISSN: 2376-7839.

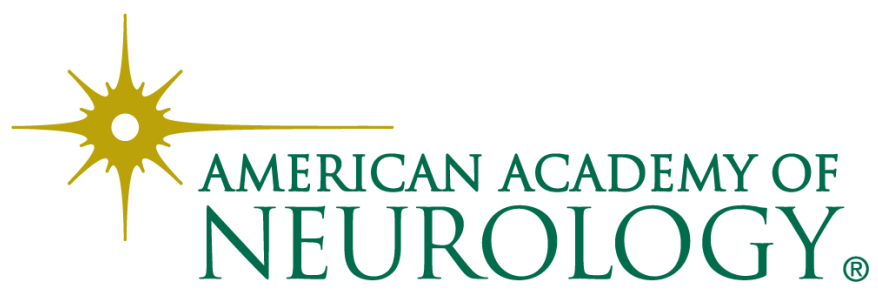

